\title{
Charge dynamics in transparent single-walled carbon nanotube films from optical transmission measurements
}

\author{
F. Borondics and K. Kamarás* \\ Research Institute for Solid State Physics and Optics, Hungarian Academy of Sciences, P.O. Box 49, Budapest 1525, Hungary \\ M. Nikolou, ${ }^{\dagger}$ D. B. Tanner, Z. H. Chen, ${ }^{\dagger}$ and A. G. Rinzler \\ Department of Physics, University of Florida, Gainesville, Florida 32611, USA
}

(Received 30 March 2006; published 31 July 2006)

\begin{abstract}
We report on the transmission over a wide frequency range (from the far infrared through the visible) of pristine and hole-doped, free-standing carbon nanotube films at temperatures between 50 and $300 \mathrm{~K}$. Optical constants are estimated by Kramers-Kronig analysis of the transmittance. We see evidence in the far infrared for a gap below $10 \mathrm{meV}$. Hole doping causes a shift of spectral weight from the first interband transition into the far infrared. Temperature dependence in both the doped and undoped samples is restricted to the farinfrared region.
\end{abstract}

DOI: 10.1103/PhysRevB.74.045431

PACS number(s): 73.63.Fg, 78.67.Ch, 81.07.De

\section{INTRODUCTION}

As carbon nanotubes attract more and more attention for use as electronic materials, so grows the need for the accurate determination of their fundamental electrical and optical properties. However, for practical reasons, these properties are not easy to determine. The optical response of a carbon nanotube depends on the tube diameter, chirality, and orientation. Bulk samples, as well as thin films, are made up of many tube types which differ in the values of these parameters. In addition the samples have a rough surface, contain void space, and have variability regarding the interaction between tubes, e.g., the strength of intertube hopping. Despite these less than ideal properties, applications exist where single-walled carbon nanotube (SWNT) thin films offer superior performance to other materials. ${ }^{1}$ In this paper we present the frequency-dependent optical functions determined from transmission through high-quality free-standing nanotube films in a wide frequency and temperature range, followed by Kramers-Kronig analysis of the transmittance.

Nanotube optical properties have been studied by a number of researchers. Some studies ${ }^{2,3}$ have been restricted to photon energies between $0.5-3 \mathrm{eV}$. Other measurements ${ }^{4-6}$ have included lower frequencies, where metallic tubes exhibit free-carrier absorption. Studies in the low frequency range also allow one to test the prediction that certain tubes would have very small gaps. ${ }^{7-9}$ These gaps have been reported near $10 \mathrm{meV}$, with substantial variation from sample to sample. ${ }^{4-6}$

Our measurements improve on earlier reports ${ }^{4-6,10-12}$ in several ways. Thick films used for reflectance measurements ${ }^{4,5,10}$ had poor surface quality. Transmission of films deposited on substrates ${ }^{6}$ required the use of several different substrate materials, making normalization difficult; moreover, temperature studies in this configuration are hindered by the thermal properties of the substrates. Transmission studies ${ }^{11}$ of free-standing nanotube films found evidence for free-carrier response down to $50 \mathrm{meV}$ but were not analyzed for optical constants. We present here measurements over a wide frequency range on free-standing samples, studying both the temperature dependence and the redistribution of carriers upon doping.

\section{EXPERIMENTAL PROCEDURES}

Film preparation, starting from laser-ablated SWNT's of $1.37 \mathrm{~nm}$ mean diameter, ${ }^{13}$ was described in Ref. 1 . Details of processing for the samples are given in Table I. The sample thicknesses were determined by atomic force microscopy (AFM) measurements. Samples A and C were measured as prepared ("unbaked"). Thermal processing ("baking") consisted of treating the samples in flowing pure argon at $1000{ }^{\circ} \mathrm{C}$ for $12 \mathrm{~h}$, to remove from the sample the nitric acid, used during purification. ${ }^{6,12}$ Sample $\mathrm{B}$ was a second piece of the same film as sample $A$; samples $C$ and $C^{\prime}$ were the same piece of film measured both before and after baking. Spectra were taken on three different spectrometers with sufficient spectral overlap to allow unambiguous merging of transmittance curves: a Bruker 113v Fourier-transform interferometer $\left(20-3000 \mathrm{~cm}^{-1}\right)$ with a Si bolometer (FIR) and a DTGS detector (MIR), a homemade near infrared/visible/UV spectrometer based on a Perkin-Elmer monochromator (2000-40 $000 \mathrm{~cm}^{-1}$ ), and a Perkin-Elmer Lambda 900 VIS/UV spectrometer. Temperature-dependent measurements were conducted with the first two instruments in a flowthrough $\mathrm{He}$ cryostat with polyethylene and $\mathrm{KBr}$ windows, respectively. The polyethylene windows caused interference fringes in the transmission spectra which, however,

TABLE I. Processing details of the samples studied.

\begin{tabular}{cccc}
\hline \hline & & \multicolumn{2}{c}{ Processing } \\
\cline { 3 - 4 } ID & Thickness $(\mathrm{nm})$ & Temperature $\left({ }^{\circ} \mathrm{C}\right)$ & Time $(\mathrm{h})$ \\
\hline $\mathrm{A}$ & 250 & as-prepared & \\
$\mathrm{B}$ & 250 & 1000 & 12 \\
$\mathrm{C}$ & 150 & as-prepared & 12 \\
$\mathrm{C}^{\prime}$ & 150 & 1000 & 12 \\
\hline \hline
\end{tabular}


do not change the level of transmission. We used a transparent sample of $5 \mathrm{~mm}$ diameter, producing enough signal even at the lowest frequencies where transmission was low. The spectra were reproducible during several cooling and heating cycles.

In the usual analysis of visible/near-IR spectra, one calculates the extinction $(-\ln T$ or $-\log T)$ from the transmission and calls this quantity the absorbance, or the product of absorption coefficient times thickness, without making further corrections. For many materials this method is perfectly adequate, as the amount of light reflected by the sample is small and only weakly dependent on wavelength. However, this procedure is not justified for strongly absorbing materials; in these the reflection (and its wavelength dependence) is a major factor, indeed often the dominant one, in determining the light transmission. This situation is the case in nanotube samples at low frequencies. Therefore, we determined the optical functions by Kramers-Kronig analysis of the transmittance.

Kramers-Kronig analysis is not as commonly applied to transmittance as it is to reflectance. Nevertheless, the transmittance of a film is subject to the same causality restrictions as the reflectance; consequently, one may estimate the phase shift on transmittance from a Kramers-Kronig integral, much as one does for reflectance. The procedure requires freestanding, uniform-thickness films, wide spectral coverage, and reasonable photometric accuracy, $\mathcal{O}(1 \%)$. The relationship between the phase shift $\Theta(\omega)$ and the transmittance $T(\omega)$ may be written in the following illuminating form:

$$
\Theta(\omega)=-\frac{1}{2 \pi} \int_{0}^{\infty} \ln \frac{|s+\omega|}{|s-\omega|} \frac{d \ln T(s)}{d s} d s .
$$

According to Eq. (1), spectral regions in which the transmittance is constant do not contribute to the integral. Further, the spectral regions $s \gg \omega$ and $s \ll \omega$ do not contribute much because the function $\ln |(s+\omega) /(s-\omega)|$ is small in these regions.

Formally, the phase-shift integral requires knowledge of the transmittance at all frequencies. In practice, one obtains the transmittance over as wide a frequency range as possible and then completes the transform by extrapolating the transmittance to frequencies above and below the range of the available measurements. The conventional low-frequency extrapolation for metals is $T(\omega)=T(0)+A \omega^{2}$, where $A$ is a constant determined by the transmittance at the lowest frequency measured in the experiment and $T(0)$ is the extrapolated behavior to zero frequency, determined by the dc conductivity. The high-frequency extrapolation uses $T=1$ $-C \omega^{-n}$ with $n \approx 1$ and $C$ chosen to give a smooth connection to the high-frequency transmittance curve.

After computing the phase, one may extract the complex refractive index $N$ (and all other optical constants) by numerical solution of (Ref. 14)

$$
\sqrt{T} e^{i \Theta}=\frac{4 N}{(N+1)^{2} e^{-i \delta}-(N-1)^{2} e^{i \delta}},
$$

where $\delta=\omega N d / c$ with $d$ the film thickness. An important detail is that the phase gained by the radiation in passing

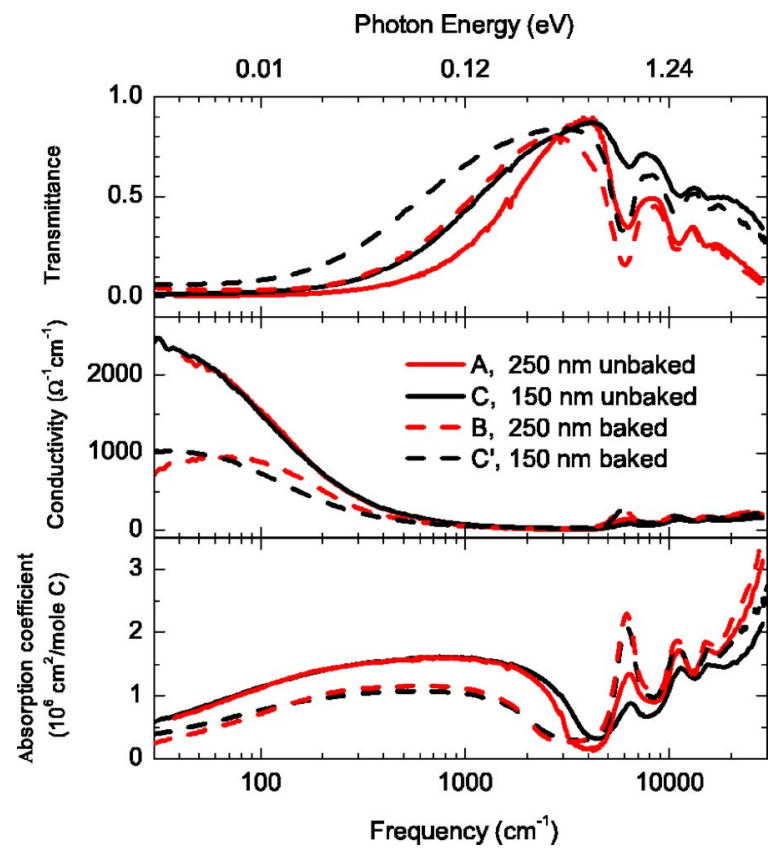

FIG. 1. (Color online) Transmittance, optical conductivity, and absorption coefficient of two SWNT transparent films of different thickness.

through a thickness $d$ of vacuum must be added to $\delta$ before calculating $N$. Equation (2) includes, of course, interfacial reflections, including multiple reflections within the sample.

\section{RESULTS}

Figure 1 shows the room-temperature transmission, optical conductivity, and calculated absorbance spectra before and after baking. Above $4000 \mathrm{~cm}^{-1}$, the absorption coefficients calculated from the Kramers-Kronig transformation agree with $(-\ln T)$.

The absorption coefficient of the material is the absorbance per unit thickness; to obtain absorption coefficients in units of $\mathrm{cm}^{2} /($ mole $\mathrm{C})$, we have to divide by the density. We determined the density of our materials directly by weighing a piece whose thickness was measured by atomic force microscopy, and obtained $0.4 \mathrm{~g} / \mathrm{cm}^{3}$. Our absorption values are higher than those found by Zhao et al..$^{15}$ in a comprehensive study of various nanotubes. They found $\alpha\left(S_{22}\right)$ of $0.6-1.1 \cdot 10^{6}$ (in our units), compared with our value (in the baked samples) of $1.8-1.9 \cdot 10^{6}$. One reason for the difference may be that in our films the long axes of the tubes are largely aligned in the plane of the film, whereas in solution spectroscopy they are disordered in three dimensions.

We also performed a Drude-Lorentz fit to the transmission curves, using the Airy formula ${ }^{14}$ for the transmittance of a thin, free-standing film. Table II lists the fitting parameters obtained. The fits and the contributions of individual oscillators to the optical conductivity at $300 \mathrm{~K}$ are illustrated in Fig. 2. We used only electronic oscillators to model the spectra; no vibrational transitions are discernible in the baked samples, even after the baseline correction described recently by Kim et al. ${ }^{16}$ In the doped materials, we observed the weak 
TABLE II. Drude-Lorentz fit parameters [plasma frequency $\left(\omega_{p i}\right)$, center frequency $\left(\omega_{i}\right)$, and width $\left(\gamma_{i}\right)$ in $\mathrm{cm}^{-1}$ ] for each sample studied at several temperatures. For peak 0, the free-carrier (Drude) absorption, the center frequency is 0. The starting parameters for high-frequency oscillators were taken from Ref. 17. The high-frequency dielectric constant is 1.04 .

\begin{tabular}{lccccccccccccccc}
\hline \hline & & $\omega_{p 0}$ & $\gamma_{0}$ & $\omega_{p 1}$ & $\omega_{1}$ & $\gamma_{1}$ & $\omega_{p 2}$ & $\omega_{2}$ & $\gamma_{2}$ & $\omega_{p 3}$ & $\omega_{3}$ & $\gamma_{3}$ & $\omega_{p 4}$ & $\omega_{4}$ & $\gamma_{4}$ \\
\hline Sample A & $300 \mathrm{~K}$ & 2057 & 762 & 4055 & 35 & 128 & 3419 & 6189 & 1435 & 5268 & 10616 & 3888 & 2505 & 14884 & 2199 \\
Doped & & & & & & & & & & & & & & \\
Sample B & $50 \mathrm{~K}$ & 1817 & 2555 & 3257 & 58 & 154 & 4613 & 5913 & 1298 & 4105 & 10429 & 2587 & 1689 & 14763 & 1310 \\
De-doped & $100 \mathrm{~K}$ & 1927 & 2661 & 3258 & 56 & 158 & 4615 & 5911 & 1308 & 4079 & 10436 & 2552 & 1721 & 14771 & 1342 \\
& $200 \mathrm{~K}$ & 1863 & 2250 & 3242 & 65 & 166 & 4573 & 5913 & 1277 & 4085 & 10424 & 2597 & 1676 & 14764 & 1306 \\
& $300 \mathrm{~K}$ & 1926 & 2232 & 3226 & 66 & 187 & 4600 & 5910 & 1310 & 4076 & 10435 & 2571 & 1725 & 14772 & 1355 \\
Sample C & $100 \mathrm{~K}$ & 2314 & 3393 & 4408 & 0 & 134 & 2296 & 6245 & 1648 & 4074 & 10859 & 3591 & 2674 & 15400 & 3880 \\
Most doped & $200 \mathrm{~K}$ & 2318 & 2944 & 4381 & 0 & 139 & 2318 & 6241 & 1679 & 4083 & 10868 & 3603 & 2646 & 15402 & 3802 \\
& $300 \mathrm{~K}$ & 2302 & 2509 & 4337 & 0 & 144 & 2328 & 6244 & 1655 & 4114 & 10858 & 3624 & 2646 & 15380 & 3793 \\
Sample C' & $100 \mathrm{~K}$ & 1984 & 2136 & 2904 & 20 & 122 & 4044 & 5937 & 1176 & 4459 & 10442 & 3122 & 1937 & 15136 & 1977 \\
De-doped & $200 \mathrm{~K}$ & 2220 & 2307 & 2917 & 20 & 131 & 4037 & 5919 & 1162 & 4502 & 10410 & 3187 & 1975 & 15194 & 2014 \\
& $300 \mathrm{~K}$ & 2561 & 2442 & 2917 & 20 & 140 & 4206 & 5935 & 1230 & 4520 & 10436 & 3160 & 1756 & 15194 & 1585 \\
\hline \hline
\end{tabular}

vibrational peaks seen by Hennrich et al. $;^{12}$ these disappear on baking.

The conductivity and the absorbance are independent of thickness except at very low frequencies; even there, the values are comparable.

\section{DISCUSSION}

\section{A. Baked (undoped) samples}

We discuss first the room-temperature results on the baked samples, because we regard these as closest to pristine carbon nanotubes. Optical spectroscopy is routinely used for purity evaluation of nanotube samples ${ }^{15,18}$ where the amount of amorphous carbon is deduced from the background absorption in the near infrared. Comparing our spectra with those by Zhao et al. ${ }^{15}$ we estimate the purity to equal those of their best samples. We assume the films dense and iso- tropic enough to apply a model of a continous medium. (Effective-medium models have been employed for polarized measurements where the shape of the tubes is crucial. ${ }^{19}$ )

The low-frequency conductivity can be best described by a two-component model. In sample B, the two components consist of a Drude free-electron contribution and a Lorentzian peak centered near $0.01 \mathrm{eV}$. In sample $\mathrm{C}^{\prime}$, we found that a fit with two Drude contributions, with distinct damping constants, also described the data. We note, however, that if the center frequency of the Lorentzian shifts below the measured range $\left(\sim 30 \mathrm{~cm}^{-1}\right)$, then it is essentially indistinguishable from a Drude curve. Indeed, an equally good fit is obtained if we fix the frequency of this peak at $20 \mathrm{~cm}^{-1}$ (close to that observed by terahertz spectroscopy ${ }^{19}$ ). We can also compare our data to those of Hilt et al. ${ }^{20}$ measured at terahertz frequencies in similar laser-ablated nanotubes as used in the present study. Although the exact doping level of the tubes studied there is not known, they also find a narrow

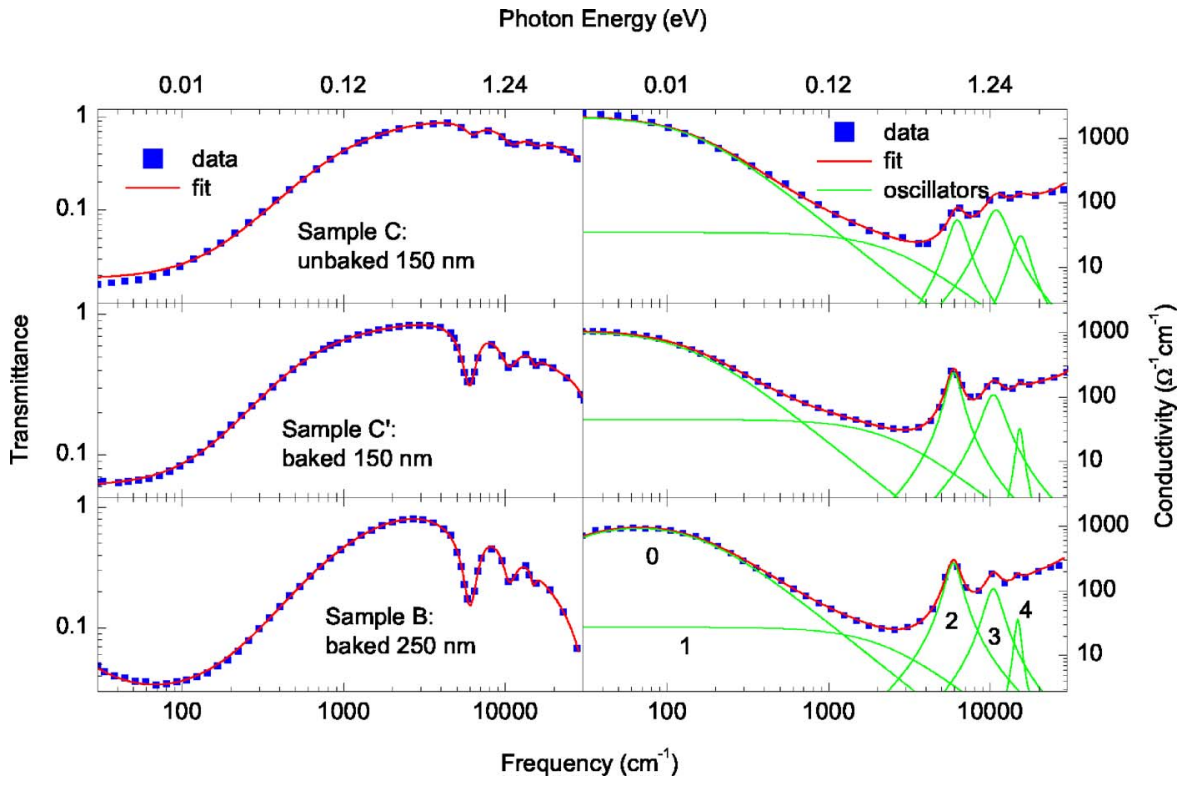

FIG. 2. (Color online) The results of Drude-Lorentz fits (leastsquares fit to transmission curves) compared to measured room-temperature transmission data and to conductivity obtained by Kramers-Kronig transformation. Numbers refer to oscillator labels in Table II. 


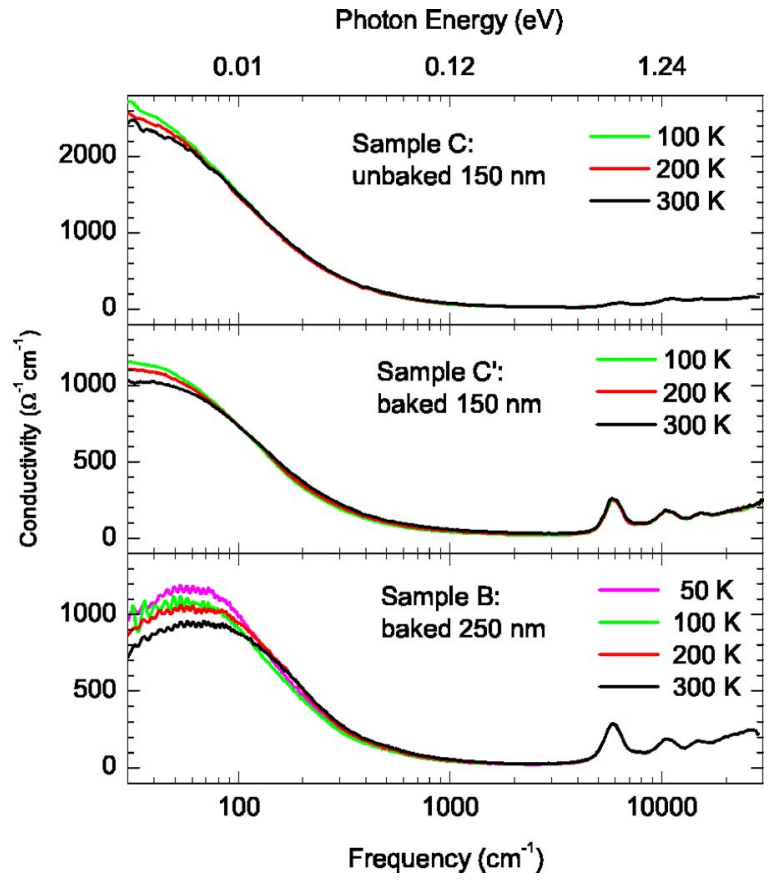

FIG. 3. (Color online) Temperature dependence of the optical conductivity of the two samples.

Drude component and a broad absorption, modeled as a constant background conductivity in their restricted frequency range.

Thus we assign the Drude part to truly metallic tubes and the low-frequency Lorentzian to the curvature-induced gap suggested by Kane and $\mathrm{Mele}^{7}$ and observed in individual nanotubes by scanning tunneling spectroscopy. ${ }^{21}$ In our samples, this gap actually represents an average of gaps for "semimetallic" tubes of different diameter and chirality. ${ }^{22}$ Note that the width $(\gamma)$ of the Drude contribution is typically more than ten times the width of the low-energy Lorentzian, suggesting that the mobility of carriers in semiconducting tubes is much higher than in metallic tubes.

The origin of the variation among samples can be attributed to unintentional doping by atmospheric oxygen. ${ }^{23,24} \mathrm{~A}$ modest number of extra holes is enough to smear out the gaps of some of the nanotubes and change their distribution, shifting the median to lower energies. The fact that the thinner film (sample $\mathrm{C}^{\prime}$ ) appears more doped is in accordance with observations by Collins et al. ${ }^{24}$ We see no indication of the bundle-induced pseudogap at $0.1 \mathrm{eV}$ seen in Ref. 21. The disappearing of the pseudogap was predicted by Maarouf et al. ${ }^{9}$ for macroscopic samples with compositional disorder.

The temperature dependence of the conductivity is much weaker than that of a simple metal (see Fig. 3). It is confined largely to the far infrared. The low-frequency conductivity decreases with increasing temperature; a crossover at $120 \mathrm{~cm}^{-1}$ reverses this dependence up to about $2000 \mathrm{~cm}^{-1}$, where it becomes too weak to be observed. The fits to a dielectric function model (Table II) show a slight broadening of peak 1, along with a blueshift of the low-frequency peak in sample B. We saw no indication down to $50 \mathrm{~K}$ for a sudden conductivity drop, which is expected if a Luttinger liquid state develops. ${ }^{25}$

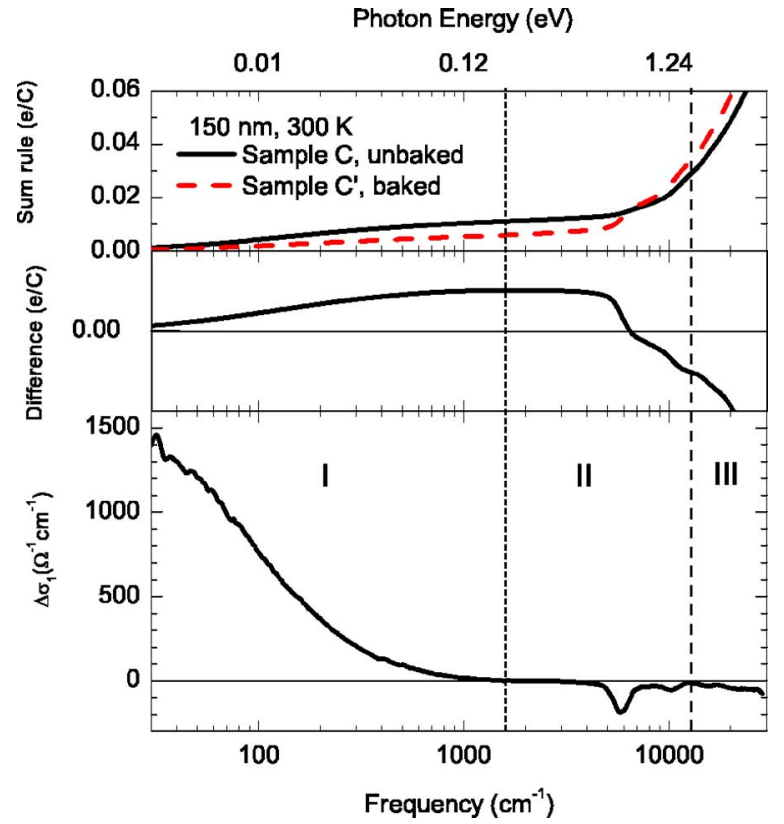

FIG. 4. (Color online) Top panel: sum rule for baked and unbaked sample at $300 \mathrm{~K}$. Middle panel: added carriers per carbon as function of frequency at selected temperatures. Bottom panel: difference in conductivity upon doping. For the definition of regions I, II, and III, see text.

The low-frequency optical conductivity of the baked samples, 800 and $1000 \Omega^{-1} \mathrm{~cm}^{-1}$ for samples B and $\mathrm{C}^{\prime}$, respectively, agree well with the $700 \Omega^{-1} \mathrm{~cm}^{-1}$ value determined from sheet resistance. ${ }^{1}$ The $2600 \Omega^{-1} \mathrm{~cm}^{-1} \mathrm{dc}$ conductivity of the unbaked sample is somewhat lower than that determined by direct sheet resistance measurements $\left(6700 \Omega^{-1} \mathrm{~cm}^{-1}\right)$ [and closer to the value $2900 \Omega^{-1} \mathrm{~cm}^{-1} \mathrm{ob}-$ tained by Liu et al. ${ }^{26}$ for $\mathrm{FeCl}_{3}$ doped nanotubes from electron-energy-loss spectroscopy (EELS) results] but the trend of higher conductivity in the unbaked samples is clear.

\section{B. Unbaked (doped) samples}

We now turn to the effect of doping on the optical properties. Figure 1 demonstrates that nitric acid doping increases the conductivity in the far infrared and decreases it around the first Van Hove transition. At low frequencies, the narrower band (the one at finite frequency in sample B) is much more influenced than the broad Drude part. We can directly compare the parameters of sample $\mathrm{C}$ (doped) and $\mathrm{C}^{\prime}$ (undoped): as a general trend, peak 0 and peak 1 both increase in intensity on doping, peak 1 more so than peak 0 , while peak 2 and peak 3 (the semiconductor tubes' $S_{11}$ and $S_{22}$ transitions, respectively) decrease. Peak 2 and peak 3 also shift to higher energies, as already reported by Itkis et al. ${ }^{6}$ The high-frequency changes upon doping have been reported and explained extensively. ${ }^{27,28}$ On hole doping, metallic tubes get depleted of charge and therefore a slight decrease in their contribution to the low frequency conductivity is expected. In semiconductors a redistribution of oscillator strength occurs as the highest-lying valence band loses electrons. This change in the occupation of states leads to a de- 
crease of the $S_{11}$ interband transition (peak 2) and at the same time, an increase in the low-frequency conductivity. The fact that this distribution strengthens peak 1 indicates that the doped semiconducting tubes also can be regarded as semimetals. At higher doping level, the same scenario occurs at the second interband transition.

The top panel of Fig. 4 shows the partial sum rule for samples $\mathrm{C}$ and $\mathrm{C}^{\prime}$. This quantity is obtained by integrating $\sigma_{1}(\omega)$ from $\omega=0$ to an upper frequency, and plotting the integrated weight versus the upper limit, representing the effective number of carriers per $\mathrm{C}$ atom taking part in optical transitions below the upper-limit frequency. Up to the near infrared, the hole-doped material shows a much higher weight than the de-doped one; at higher frequencies the difference changes sign. The middle and bottom panels of Fig. 4 show, respectively, the difference in spectral weight and the difference in conductivity $\Delta \sigma_{1}$ between the doped and undoped samples. The behavior may be divided into three regions: the free-carrier part (region I), the interband transition part (region II), and the $\pi$-electron excitation part (region III). The boundary between regions I and II is at $1765 \mathrm{~cm}^{-1}$, where the conductivity difference changes sign (bottom panel): we assume that if we had a series of concentrations, this point would be isosbestic. The sum rule difference in this part of the spectrum (middle panel) levels off at the value of $0.005 \mathrm{e} / \mathrm{C}$, before it starts to decrease at the $S_{11}$ transition. A second, smaller, negative peak in the conductivity can be seen at the $S_{22}$ frequency. We put the boundary between regions II and III at $12600 \mathrm{~cm}^{-1}$, where $\Delta \sigma_{1}$ approaches zero again, and the value of the sum rule reaches $-0.005 \mathrm{e} / \mathrm{C}$. We infer from this that the principal effect of hole doping is a redistribution of charge within the semiconducting nanotubes, and the doping of the metallic tubes is negligible in comparison. In region III the collective excitations of $\pi$-electrons can be found. ${ }^{29}$

\section{SUMMARY}

In summary, we have presented optical transmission in a wide frequency and temperature range of high-quality transparent single-walled carbon nanotube films. We found a gap feature below $10 \mathrm{meV}$ and associate it with the curvatureinduced gap of semimetallic tubes. We estimate the hole doping of our samples by nitric acid to cause a 0.005 e/C redistribution of charge between the free-carrier absorption and the interband transitions in semiconducting tubes and suggest that the free carriers in the doped semiconductors behave similarly to those in semimetallic tubes.

\section{ACKNOWLEDGMENTS}

We thank T. Pichler for enlightening discussions. This work is supported by OTKA T049338 and jointly by NSFDMR-0305043 and DOE DE-AI02-03ER46070.
*Electronic address: kamaras@ @zfki.hu

†Present address: Cornell Center for Materials Research, Cornell University, Ithaca, NY 14853, U.S.A.

Present address: IBM T. J. Watson Center, P.O. Box 218, Yorktown Heights, NY 10598, U.S.A.

${ }^{1}$ Z. Wu, Z. Chen, X. Du, J. Logan, J. Sippel, M. Nikolou, K. Kamarás, J. R. Reynolds, D. B. Tanner, A. F. Hebard, and A. G. Rinzler, Science 305, 1273 (2004).

${ }^{2}$ H. Kataura, J. Kumazawa, Y. Maniwa, I. Umezu, S. Suzuki, Y. Ohtsuka, and Y. Achiba, Synth. Met. 103, 2555 (1999).

${ }^{3}$ M. J. O'Connell, S. M. Bachilo, C. B. Huffmann, V. C. Moore, M. S. Strano, E. H. Haroz, K. L. Rialon, P. J. Boul, W. H. Noon, C. Kittrell, J. Ma, R. H. Hauge, R. B. Weissman, and R. E. Smalley, Science 297, 593 (2002).

${ }^{4}$ A. Ugawa, A. G. Rinzler, and D. B. Tanner, Phys. Rev. B 60, R11305 (1999).

${ }^{5}$ A. Ugawa, J. Hwang, H. H. Gommans, H. Tashiro, A. G. Rinzler, and D. B. Tanner, Curr. Appl. Phys. 1, 45 (2001).

${ }^{6}$ M. E. Itkis, S. Niyogi, M. E. Meng, M. A. Hamon, H. Hu, and R. C. Haddon, Nano Lett. 2, 155 (2002).

${ }^{7}$ C. L. Kane and E. J. Mele, Phys. Rev. Lett. 78, 1932 (1997).

${ }^{8}$ P. Delaney, H. J. Choi, J. Ihm, S. G. Louie, and M. L. Cohen, Nature (London) 391, 466 (1998).

${ }^{9}$ A. A. Maarouf, C. L. Kane, and E. J. Mele, Phys. Rev. B 61, 11156 (2000).

${ }^{10}$ J. Hwang, H. H. Gommans, A. Ugawa, H. Tashiro, R. Haggenmueller, K. I. Winey, J. E. Fischer, D. B. Tanner, and A. G. Rinzler, Phys. Rev. B 62, R13310 (2000).
${ }^{11}$ F. Hennrich, S. Lebedkin, S. Malik, J. Tracy, M. Barczewski, H. Rösner, and M. Kappes, Phys. Chem. Chem. Phys. 4, 2273 (2002).

${ }^{12}$ F. Hennrich, R. Wellmann, S. Malik, S. Lebedkin, and M. M. Kappes, Phys. Chem. Chem. Phys. 5, 178 (2003).

${ }^{13}$ A. G. Rinzler, J. Liu, H. Dai, P. Nikolaev, C. B. Huffmann, F. J. Rodriguez-Macías, P. J. Boul, A. H. Lu, D. Heymann, D. T. Colbert, R. S. Lee, J. E. Fischer, A. M. Rao, P. C. Eklund, and R. E. Smalley, Appl. Phys. A 67, 29 (1998).

${ }^{14} \mathrm{M}$. Dressel and G. Grüner, Electrodynamics of Solids (Cambridge University Press, Cambridge, 2002).

${ }^{15}$ B. Zhao, M. E. Itkis, S. Niyogi, H. Hu, J. Zhang, and R. C. Haddon, J. Phys. Chem. B 108, 8136 (2004).

${ }^{16}$ U. J. Kim, X. M. Liu, C. A. Furtado, G. Chen, R. Saito, J. Jiang, M. S. Dresselhaus, and P. C. Eklund, Phys. Rev. Lett. 95, 157402 (2005).

${ }^{17}$ Y. Murakami, E. Einarsson, T. Edamura, and S. Maruyama, Phys. Rev. Lett. 94, 087402 (2005).

${ }^{18}$ M. E. Itkis, D. E. Perea, S. Niyogi, S. M. Rickard, M. A. Hamon, H. Hu, B. Zhao, and R. C. Haddon, Nano Lett. 3, 309 (2003).

${ }^{19}$ T.-I. Jeon, K.-J. Kim, C. Kang, I. H. Maeng, J.-H. Son, K. H. An, J. Y. Lee, and Y. H. Lee, J. Appl. Phys. 95, 5736 (2004).

${ }^{20}$ O. Hilt, H. B. Brom, and M. Ahlskog, Phys. Rev. B 61, R5129 (2000).

${ }^{21}$ M. Ouyang, J.-L. Huang, C. L. Cheung, and C. M. Lieber, Science 292, 702 (2001).

${ }^{22}$ A. Ugawa, A. G. Rinzler, and D. B. Tanner, Ferroelectrics 249, 145 (2001). 
${ }^{23}$ G. U. Sumanasekera, C. K. W. Adu, S. Fang, and P. C. Eklund, Phys. Rev. Lett. 85, 1096 (2000).

${ }^{24}$ P. G. Collins, K. Bradley, M. Ishigami, and A. Zettl, Science 287, 1801 (2000).

${ }^{25}$ H. Ishii, H. Kataura, H. Shiozawa, H. Yoshioka, H. Otsubo, Y. Takayama, T. Miyahara, S. Suzuki, Y. Achiba, M. Nakataka, T. Narimura, M. Higashiguchi, K. Shimada, H. Namatame, and M. Taniguchi, Nature (London) 426, 540 (2003).
${ }^{26}$ X. Liu, T. Pichler, M. Knupfer, J. Fink, and H. Kataura, Phys. Rev. B 70, 205405 (2004).

${ }^{27}$ S. Kazaoui, N. Minami, R. Jacquemin, H. Kataura, and Y. Achiba, Phys. Rev. B 60, 13339 (1999).

${ }^{28}$ P. Petit, C. Mathis, C. Journet, and P. Bernier, Chem. Phys. Lett. 305, 370 (1999).

${ }^{29}$ T. Pichler, M. Sing, M. Knupfer, M. S. Golden, and J. Fink, Solid State Commun. 109, 721 (1999). 